\title{
Research on Energy-Aware Topology Strategy Based on Wireless Sensor in Internet of Things
}

\author{
XueFei Tang \\ School of Computer Science \& Engineering \\ Chengdu, 611731, China \\ XinZheng Niu \\ Chengdu, 611731, China \\ Email: xinzhengniu@uestc.edu.cn \\ Shahzad Ali \\ Bahawalpur, 63100, Pakistan \\ Email: rsdcsiub@hotmail.com
}

Received 31 July 2013

Accepted 17 August 2013

\begin{abstract}
Since the finitude of hardware and network dynamic limit the bandwidth based on wireless sensor in the wireless internet of things (IOT), it is difficult to obtain the network topology information accurately and timely. In this paper, we present an algorithm called Mobile Agent Fuzzy Energy Spanning Tree (MAFEST) for topology discovery and topology control. In the construction of IOT wireless sensor based network topology, the strategy considers the energy consumption of communication between wireless sensors, the uncertainty of agent knowledge and the sensor residual energy. This algorithm can access to the network topology information accurately and timely, and improve the efficiency of network topology discovery.
\end{abstract}

Keywords: Energy Aware, Internet of Things, Mobile Agent, Topology Strategy, Wireless Sensor

\section{Introduction}

Everything can have a connection with Internet of Things(IOT) to exchange, communicate and share the information by using the technology of radio frequency identification (RFID), infrared sensor, global positioning system and some information sensing devices. Just as the research1 has mentioned, the sensor, which is the bottom infrastructure in the IOT hierarchical structure, can be used to collect and control information. IOT is constituted by tiny and high cost performance sensor devices, which are the basis of IOT to build a relationship between physical and digital world.
For any IOT related research, acquiring the information which includes the location of sensors, the knowledge of network topology and so on will be critical to other IOT applications. Due to the influence from some hardware defects rooted in sensor itself and some other network factors, the network information that we get from the sensor nodes will be dynamic and far different from the actual situation of network which may lead to many mistakes of the network knowledge that will weaken the function of other applications. Therefore, to acquire the knowledge of sensor based network topology fast and accurately, which is a kernel of the network topology discovery of the Internet of 
IOT now, is difficult by using network monitoring tools and some robust fault tolerance technologies.

Currently, a lot of researches are being managed by some IOT research institutes. For mobile agents have characteristics of self-study, distribution, mobility and self-organization, they have been widely used in distributed sensor network, congestion control of mobile network and topology discovery etc ${ }^{2}$. Simultaneously, just as the research ${ }^{3}$ has mentioned, compared with traditional $\mathrm{C} / \mathrm{S}$ model, mobile agent is more suitable for wireless sensor network in topology discovery and data fusion etc. So this paper will focus on the problem we have mentioned above, use mobile agent-distributed computing technologies and propose a design scheme of the fuzzy and dynamic topology discovery algorithm based on mobile agents. The algorithm designs the network topology strategy according to the finding speed, accuracy and the energy consumption of network etc, which can make the network topology discovery of IOT robust and stable.

\section{Related Work}

Because the infrastructure of IOT is consist of wireless sensors which will be affected by some factors like geographic environment and network dynamic, so the network topology based on wireless sensors is dynamic and complicated. The knowledge of network topology always has a big difference from those of the actual network environment which may lead to a wrong awareness of the topology of network. As the papers3, 9 describe, there is some delay of network, overloaded amount of communication and weak scalability and robustness in network topology discovery. Also, the unbalanced energy of sensors is common and serious. Besides, in the IOT composed by a large number of sensors, the network topology can be often affected by the sensors failure and some other special elements.

\subsection{Network Topology Control}

Topology Strategy in Mobile or Wireless Networks a. Network Dynamic Element

The research ${ }^{18}$ propose a location-aided topology discovery algorithm (LAD). But the dynamic of network will affect the efficiency of the topology ${ }^{18-19}$. The paper $^{20}$ take frequent topology changes into consideration and the research fruits can optimize the topology discovery.

\section{b. Core Work of Research}

The core work of some research fruits as following aren't the topology discovery service. They have designed a density-aware GPSR routing protocol (DAGPSR) using the parameters provided by the topology discovery service, so they focus on improving the performance of the original GPSR protocol ${ }^{14}$.

\section{c. Other Mechanisms Dependency}

These topology discovery algorithms need other algorithms or mechanisms to establish efficient topology discovery services. The topology learning and collecting process, together with the used scheduling scheme, are essential parts in the design of such MAC protocols. But the proposed protocol controls the time during which CSMA is used for control message transmission in the paper ${ }^{15}$. Researchers ${ }^{16}$ propose their algorithm called Bounded Degree Energy Aware Algorithm (BDEA) which selects clusterheads by weighted clustering approach. They present a strategy for topology discovery and reconfiguration in Ad Hoc wireless sensor networks based on clusterheads' design. In this protocol ${ }^{17}$, only clusterheads will generate routing update packets as opposed to ordinary link state protocols which all nodes do so. They ${ }^{21}$ have described an algorithm for weighted topology discovery which uses colors based approach to partition the network into clusters. Each cluster has a clusterhead.

\section{Energy-Aware Topology Strategy}

Through existing research outcomes, researchers also show a number of algorithms used in constructing the network topology structure of power saving in mobile constrained wireless networks, such as CBTC, LMST and CONNECT etc. Ramanathan ${ }^{4}$ raises two centralized algorithms as CONNECT and BICONNAUGMENT when each is a simple greedy algorithm which can minimize the maximum power of nodes while maintaining the network nodes connected. The basis of their algorithms is the connection of nodes. COMPOW $^{5}$ and CLUSTERPOW ${ }^{6}$ are two algorithms implemented in the network layer which can maintain the minimum common power to keep network connectivity and extend the network lifetime, mitigate the MAC level collision. $\mathrm{CBTC}^{7}$ is a cone-based distributed topology control algorithm. It proves that $5 \pi / 6$ is sufficient for every cone of degree in every node to control network connection. $\mathrm{LMST}^{8}$ algorithm is a topology control algorithm which constructs the local minimum spanning tree based on local information. It 
can effectively reduce the overall energy consumption and mitigate the MAC level interference. However, this algorithm has some weakness. It does not take the energy-balance into consideration and may cause the "bottleneck" nodes to use up their power due to the overload of receiving packets.

The current other work of topology discovery for sensor network ${ }^{10-12}$ is energy measurement and management for the rate of packet loss, which can be done based on known network logic topology. There is no specialized way to obtain the topology of network.

\subsection{Mobile Agent System}

As a mobile agent have self-learning, distribution, mobility and self-organization features and capabilities, it is widely used in distributed sensor networks, mobile networks, congestion control, and topology discovery etc $^{2,3,9}$. As paper ${ }^{9}$ has described, based on a mobile multi-agent cooperation model, analysis of the uncertainty and influence of the agent knowledge or agent interaction has become one of the core tasks in a mobile agent based system. On the research about agent cooperation system ${ }^{13}$, we know that the knowledge is inconsistent and uncertain. We can combine these features with fuzzy theory and propose a multi-agent cooperation system. Besides, agent environmental awareness is a subjective understanding which can be divided into two categories: direct facts acquired by agent movements and indirect facts gained by exchanging information among agents. In the process of agents' movement, collaboration system can gradually build the network topology awareness, interaction and degradation over time.

Furthermore, this paper ${ }^{22}$ provides an insight into a design of mobile agent based network topology discovery system using IBM Aglets. Also, it presents their experience with an experimental approach rather than a more common simulation based approach while the research needs to do more important performance test. This research ${ }^{23}$ proposes to assess different models of the usage of static and mobile agents to determine the best route through Ad Hoc networks. The usability of the approach is proved by an implementation of mobile devices using $\mathrm{J} 2 \mathrm{ME}$ and Bluetooth ${ }^{24}$. So they aim to generate the best route through a network ${ }^{23-24}$. It is more difficult in networks where the topology changes very frequently such as mobile Ad Hoc networks. They present a mobile agent based topology discovery framework that is distributed, sustainable and less resource intensive ${ }^{25}$. This paper introduces a strategy based on mobile agents and swarm intelligence for topology discovery ${ }^{26}$ while their future work still includes devising a methodology to measure the accuracy of the topology known by the nodes at any given time ${ }^{25-26}$. The task of fault management ${ }^{27}$ is even harder in Ad Hoc networks where the topology of the network changes frequently. The paper proposes a mobile multi agent system for fault management functions of topology discovery.

In summary, based on these issues and research fruits, this paper proposes a fuzzy dynamic control strategy based on mobile agent and energy control using distributed technology of mobile agent. This strategy constructs network topology according to the knowledge of discovery speed, accuracy, and network energy consumption. It makes the topology of IOT with a high robustness and stability with properly considers and resolves key issues such as agent's fuzzy belief synthesis, knowledge contradictions processing and energy consumption balance. Finally, through experiments, this paper compares the model and algorithm with existing research fruits. The result shows that the design and analysis of the algorithm we proposed is effective and has a realistic significance of application.

\section{Description and Analysis of Model}

\subsection{Network Topology Strategy Model}

The behavior of a mobile agent is generally divided into active and passive while active mode refers that the agent discovers network topology initiatively according to the environmental condition and passive mode means that the agent's activities are controlled by other entities (such as sensor nodes). The agents of this paper will experience a proactive approach to discover network topology. They walk through the network, collect information when meeting a sensor node and exchanging knowledge with other agents. Specifically, agents get direct facts by themselves, while indirect facts through knowledge exchange. Obviously, the former information is clear and true, while the latter is fuzzy, even wrong. Because the dynamic of network, the lapse of time and the distribution of network events which will occur in the network will cause agent's undefined and fuzzy recognition of facts. Therefore, in 
this case, agent's knowledge becomes fuzzy and fuzzy knowledge constitutes agent's beliefs. Besides, agents also need to update energy information of network sensors.

\subsection{Agent Model Structure}

Mobile agent is a logic execution unit for network topology discovery of a dynamic network. According to the agent structure of Ref. 13 mentioned, we make the following definition of the model:

$$
\text { IAgent }=<M_{A}, E_{\Omega}, L_{U}, H_{R}, C_{D}, S_{G}>
$$

Where $M_{A}$ represents the mobile agent network ID;

$E_{\Omega}$ represents an information collection of overall network topology discovery, including the sensor nodes shutdown or startup, disconnect or reconnect the link, link congestion or smooth and other information.

$L_{U}$ represents the network topology discovery collection of mobile agent $i$ by exchanging information with other mobile agents. This collection is a part of network knowledge.

$$
L_{U}=\left\{l_{U 1}, l_{U 2}, \cdots, l_{U N}\right\}, N \geq 0, l_{U i} \in E_{\Omega}, L_{U} \subseteq E_{\Omega} .
$$

$H_{R}$ represents the threshold setting for agent $i$ (See fuzzy knowledge processing), which checks the network topology discovery of fuzzy beliefs and determines whether they can accept the fact to make different actions or not. $H_{R}=\{$ accept, reject, exchange, save, quit $\}$. If mobile agent $i$ accepts to use the network topology knowledge provided by IOT network sensors, then it makes an accept action, otherwise rejects it. The data, which agent $i$ needs to modify its network topology discovery information, can be transacted by exchange action. If agent $i$ has exchanged data successfully, it can use the data to modify their own fuzzy network topology by save action. When has finished the interactive process of fuzzy knowledge exchange, agent performs the quit action. Among these sensors, the sensor node, known as mobile agent environment, provides the assurance requirements for agent with basic behaviors such as transmission, interactive information and so on. There is a "blackboard" in the sensor node by which more than one agent can interact with each other indirectly. All sensor nodes will refresh the information on the blackboard while under the influence of the information. The information constitutes integrated knowledge, represents local performance of agent knowledge groups and reflects the overall network status.
$C_{D}$ represents the fuzzy belief collection of network topology discovery after agent $i$ exchange knowledge with agent $j$ or other agents. $C_{D}=\left\{C F\left(i, e_{1}, t_{1}\right), C F\left(i, e_{2}, t_{2}\right), \cdots\right\}$. Belief parameter $C F(i, e, t)$ represents the fuzzy trust of network topology discovery $e$, Where $e \in E_{\Omega}$.

$S_{G}$ represents the confidence degree of agent $i$ with network topology discovery. $S_{G}=\left\{F A\left(e_{1}\right), F A\left(e_{2}\right), \ldots\right\}$. $S_{G}$ is directly influenced by $C_{D}$. Use descriptions in the Ref. 9, we define $F A\left(e_{k}\right)$ as:

$$
F A\left(e_{k}\right)=\left|C F\left(i, e_{k}, t\right)-0.5\right|, 0 \leq F A\left(e_{k}\right) \leq 0.5
$$

\subsection{Control of Mobile Agent Activity}

Mobile agents in network will consume network bandwidth and frequent movements are prone to increase packet loss when packets of sensor nodes transmit. To control the moving rate of mobile agent in the network, the concept of mobile agent activity is proposed.

General Confidence $\overline{S_{G}}$ : It is the overall evaluation of all knowledge in the agent. We define $\overline{S_{G}}=2 \sum_{e \in E_{\Omega}} \varphi_{e} \times F A(e)$, where $\varphi_{e}=\frac{F A(e)}{\sum_{k \in E_{\Omega}} F A(k)}$ represents the weight of total belief in agents' network topology discovery $e$.

Network Activity Nac: It is an understanding level of agent for the dynamic change on the network and a function about updating time of network topology discovery, defined as $N a c=2-e^{\theta_{t}^{2}}$, where $\theta_{t}$ is the rate between recent system time difference of two network topology discovery and agent network update cycle. $\theta_{t}=$ (Current time - time for recent events) $/$ update cycle network topology discovery.

Agent Activity: It represents the moving speed of agent in network, defined as $A a c$. It is a function of $\overline{S_{G}}$ and $N a c$. With network becoming gradual stable, the activity of agent can be shown in Figure 1. We can use the following function which denotes the relationship between activity and two others.

$$
A a c=N a c \times \sqrt[3]{e^{-\overline{S_{G}}}}
$$

According to the $T_{\text {linger }}$, we can control the moving speed of a mobile agent in the network, which finally affects the network bandwidth consumption of agents. 


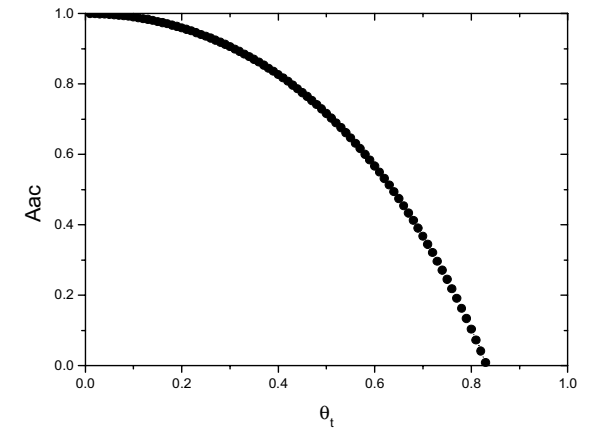

Fig. 1. Relationship between mobile agent speed and time

\subsection{Fuzzy Energy of Sensor Nodes}

Maximum communication range $d_{\max }$ : It is the communication radius of sensor nodes under the maximum transmission power.

Fuzzy network topology $G_{i}{ }^{\prime}=\left(E^{n}, F^{n}\right)$ : Every agent $i$ has a fuzzy topology $G_{i}{ }^{\prime}$ to save agent $i$ 's fuzzy energy of network. $E^{n}$ is the estimated energy of remaining sensor nodes. $F^{n}$ is a fuzzy belief of $E^{n}$.

To facilitate the description of the algorithm, we make the following assumptions: (i) Maximum transmission power is the same for each sensor node and communication radius can be changed by adjusting transmission power, (ii) The initial energy of each sensor node is the same and the position can be obtained by position algorithms or GPS location information, (iii) The relationship between energy consumption of wireless communication and communication distance ${ }^{11}$ is shown as below:

$$
E_{x}=k d(u, v)^{m}+C
$$

Where $E_{x}$ is the energy cost, $u$ is the transmitter sensor node, $v$ is the receiver sensor node, $d(u, v)$ is communication distance. $C, k$ are system constants. In IOT, $m$ is constrained by many factors. Taken together, take $m=3$.

\section{Network Topology Discovery Strategy based Energy aware and Mobile Agent - MAFEST}

Although the multi-agent system for mobile communication we researched can quickly discover the network topology ${ }^{6,7}$, the frequency of topology changes occur in the network can make its sensitivity decrease. We simulated it in an environment with 100 sensors with periodically change the topology of the network.
The result of simulation is shown in figure 2, the $\mathrm{X}$-axis represents how long does the network run. The Y-axis represents the fuzzy belief of one random sensor in this changing topology (compare the results of the paper ${ }^{9}$ ). We can see a decrease of perception happened in figure 2 (close to one straight line in the end) when changing period become $250 \mathrm{~ms}$.

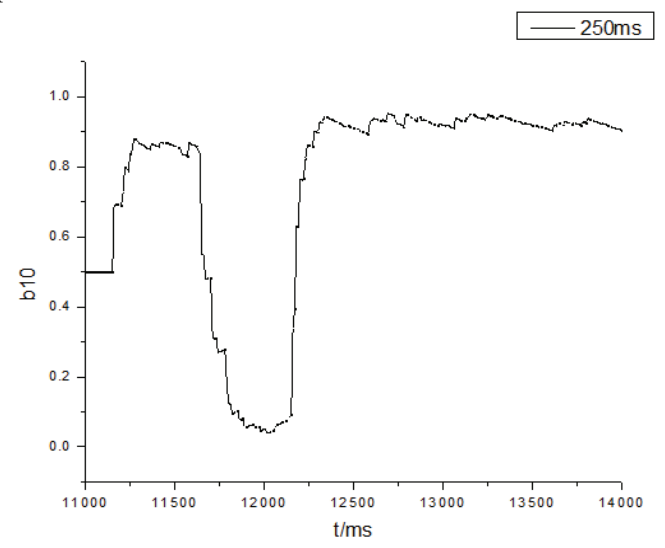

Fig. 2. Periodically topology change(every $250 \mathrm{~ms}$ )

\subsection{Algorithm formula}

Fuzzy knowledge processing

Thresholds of Belief Parameter $Z_{c f}$ : It represents if the information obtained by mobile agent or sensor node topology is available, values of belief parameter must be reached. Only when the mobile agent or belief parameters are greater than value of sensor node, topology information carried by the mobile agent or sensor can be trusted and used.

Belief Influence Effect ${ }^{9}$ : It represents the degree of agent's network topology discovery knowledge influenced by other agent or sensor's information. effect $=\sum_{k \in H} w_{k} C F\left(i, e_{k}, t\right)-w_{e} C F(i, e, t)$, where $H$ is the agent collection for information exchange, $H \subseteq M_{A}$. And $w_{e}=C F(i, e, t) / \sum_{k \in \mathrm{H}} C F(k, e, t)$, which is the belief percentage of total network topology referring to network discovery $e$ in $H$.

Energy-aware model

Energy is usually limited by the battery power of sensor node in IOT. In order to extend the working lifecycle of the entire network, balance sensors' energy needs to be considered. We propose a network energy-aware model based multi-agent system, which combines the features of sensor nodes and the ideas of topology discovery. It 
can deal with frequent changes in topology caused by situations such as energy conservation or sensor nodes' energy exhaustion.

The calculation of this algorithm's link weight takes sensor communications energy and residual energy into consideration, combining with the fuzzy knowledge discovery. We define the process of fuzzy energy belief exchange as $F^{n}=\sum F^{k} \times \tau_{k}$, where $\psi$ is the sensor node collection of whíle network, $\tau_{k}=\frac{F^{k}}{\Gamma F^{j}}$.

We define process of fuzzy energy $E_{j \in \mathcal{v}}^{2} \mathrm{exchange}^{j}$

$$
E^{n}=\left\{\begin{array}{cc}
\frac{\sum_{i \in \pi} R_{i} \times F_{i}^{n}}{\sum_{i \in \pi} F_{i}^{n}} & , n \neq m \\
R_{m} & , n=m
\end{array}\right.
$$

Where $\pi$ is the agent collection resided in sensor $n$; $m$ is network sensor number of fuzzy topology network. $R$ is the real residual energy of sensor obtained by Agent $i$.

The link weight from sensor node $u$ to node $v$ is defined as:

$$
w(u, v)=\left\{\begin{array}{cc}
\frac{d(u, v)}{d_{\max }} \times \frac{E_{x}}{E_{v}} \times \frac{2 E_{0}}{E_{u}+E_{v}} & , \frac{E_{x}}{E_{v}} \leq 1 \\
\infty & , \frac{E_{x}}{E_{v}}>1
\end{array}\right.
$$

Where $E_{x}$ is the energy consumption of wireless communication; $E_{0}$ is initial energy of sensor; $E_{x} / E_{v}<1$ means sensors can use the remaining energy to send one packet, not vice versa. $E_{u}$ is the residual energy of the sending sensor $u ; E_{v}$ is residual energy of the receiving sensor $v$. They all calculate residual energy using the energy information carried by mobile agents and gained by their own packets, where using different $\alpha, \beta$ weight value. $0 \prec \alpha \prec 1,0 \prec \beta \prec 1, \alpha+\beta=1$.

\subsection{Algorithm description}

The information agent of network gained information including the information of sensor node, which has probing packets of the network sensor node sent them to network aiming at detecting the network topology information. Different from the packet which is used in the information exchange between mobile agents, the packet is without faith and cannot identify the trusted level of information while it can be used in the agent for network topology discovery. We've designed the query message content in the network. It includes the packet type, number of packet and IDs of sensor nodes that packet has passed. Moreover, basic information of sensors that packet has passed includes each sensor's ID and the time leave the sensor.
Gathering up the above design and description, steps of MAFEST algorithm is as follows:

STEP1: Each sensor sends probing packets to neighbor sensors randomly which record the basic information of sensors it will pass by. Each network sensor node monitors the process of fuzzy knowledge exchange among the mobile agents and reconstructs the fuzzy network topology. Then update sensors' residual energy if a sensor finds out an obvious change of one sensor (or edges) in the fuzzy network topology $G^{\prime}$.

STEP2: Using mobile agents to discover topology in the network. Each mobile agent in this network uses a certain strategy for movement. Once it reaches a new sensor, it collects detection packets information. While exchanging fuzzy knowledge with other agents, it updates the network topology knowledge among all agents using the calculated value of belief effecteffect and belief parameter thresholds- $\mathrm{Z}_{c f}$. Whenever a new mobile agent reaches any sensor nodes, all mobile agents resided in this sensor should exchange knowledge with this sensor using blackboard.

STEP3: After mobile agent is wandering steadily in the network, we use Eq. (5) as a link weight between each network sensor to calculate its local minimum spanning tree. Then it adjusts power according to the local minimum spanning tree information and makes the communication radius of each sensor satisfied the demand of being able to reach all one-hop neighbor sensors around it. This is a bidirectional process about edges in addition. Furthermore, the resident time of agent in sensors $T_{\text {linger }}$ shows its linear relationship with Aac (see Eq. (2) for detail), and the belief of fuzzy energy can be calculated through Eq. (4).

STEP4: When the agent moves from one sensor to the next, firstly, they sort sensors and rank in routing table respectively according to their belief value. Then, they choose one sensor which has the minimum belief as the destination sensor with selecting the link which has the minimum belief as the path passed through, i.e., $\min F A(e), e \in E_{\Omega}$.

STEP5: Agents and sensors repeat step 1 to 4 until they exit from the network.

\section{Simulation Model}

\subsection{Simulation environment}

The simulation environment is a dynamic environment has different pairs of network sensors and edges such as 
$14 / 20,28 / 46,56 / 104$. Link capacity is $2.0 \mathrm{Mpbs}$ and simulation time period is millisecond. Assume that sensor node generates data packets which will follow Poisson distribution. The average size of data packets produced is 1024 bytes.

\subsection{Simulation results}

After comparison, we find that the response rates of network topology discovery are quite different by a different number of mobile agents in the whole network. In figure 3, Y-axis represents the percentage of sensors known the event occurred and $\mathrm{X}$-axis $(\Delta t)$ represents the time passed when the real event occur. In figure 3 with 56 network sensors, we compare the transmission speed with the number of agents such as $8,14,28, \ldots$ 224 etc. The figure 3 indicates that the number of agents can increase the speed of transmission of network discovery. On the other hand, after a certain amount of increase, if we continue to increase the total amount of agents, the discovery transmission speed will decrease.

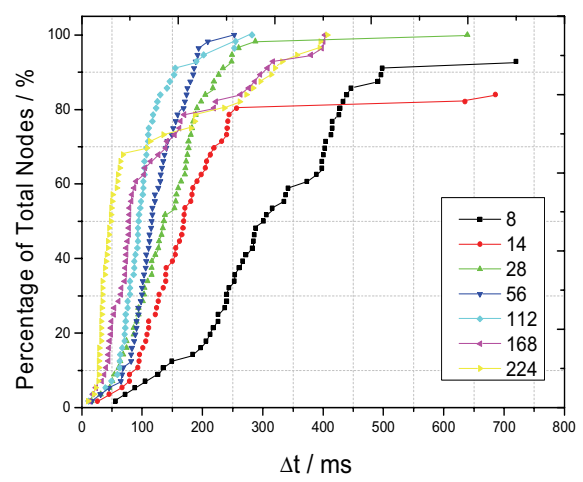

Fig. 3. (Color) Influence to transmission time with different amountof agents(The number of network sensors node: 56 )

The result in figure 4 indicates that there is a relationship between the number of agents and network sensor nodes' knowledge about the network sensitiveness. $\mathrm{X}$-axis (Rate) is the percentage of mobile agents and network sensor nodes and $\mathrm{Y}$-axis is the time passed since the network changing event occurred. While in figure 5, it describes the relationship between number of agents and network bandwidth. The result shows that the number of agents and the speed of network topology discovery are in an opposite direction while network bandwidth consuming is in a linear relationship with the number of agents. And unit is packets/s.

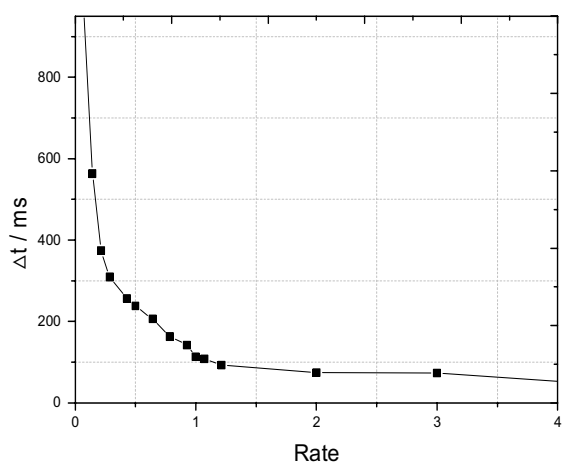

Fig. 4. Relationship between number of agents and response time

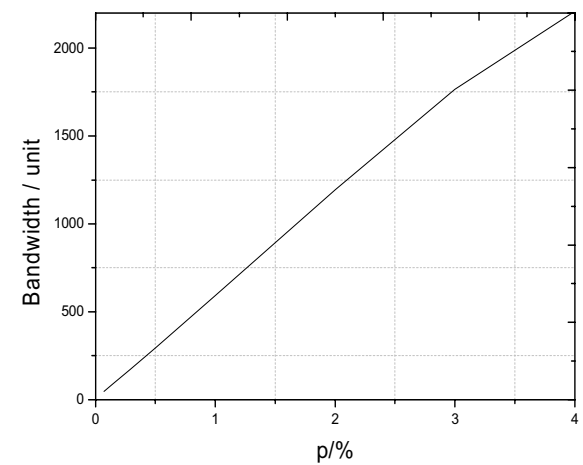

Fig. 5. Relationship between number of agents and bandwidth

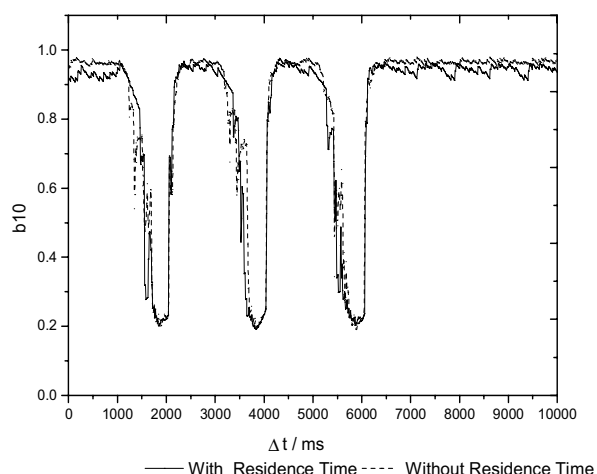

Fig. 6. Influence of residence time on system dynamic response time

Combining the figures 3 to 5 , we find that the ratio number of agents and network sensor nodes is about 
$0.5 \sim 1$ (Figure 4). The bandwidth of network is changing in the range of 250 to 600 units (Figure 5). The network discovery transmission speed is about $200 \mathrm{~ms}$ which is acceptable for us in the practical application. Moreover, we verified the influence to network bandwidth using agent activities and fuzzy knowledge disposal in the algorithm above. If the movement of agents between sensor nodes is frequent, it will lead to network congestion and prone to the loss of data packet in transmission. By increasing the residence time of agents in one sensor, we can control network bandwidth effective utility rate and analyze the speed of agents' movement to optimize the response time of network discovery.

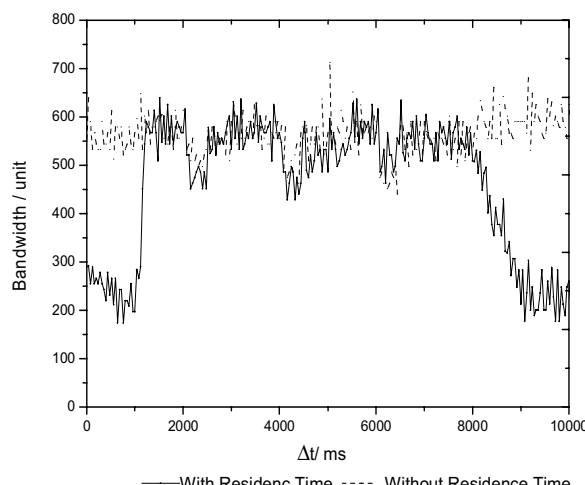

Fig. 7.Relationship between time and network bandwidth

In figure 6 , the increases or decreases of fuzzy value indicate a network event occur in network topology discovery, such as link crash or reconnection. The increased time of agent residence time won't affect the agents' speed of network topology discovery (For example fuzzy belief design, see Ref.9 for more detail). And figure 7 indicates that agents can increase their speed when the network becomes active to find the change of topology. It indicates that when network becomes active, the new strategy can get a timely response to the event of topology change, while reducing the activity of agents to reduce network bandwidth when network become stable.

In this paper, MAFEST algorithm we proposed also takes the residual energy of each sensor into account, using the fuzzy concept of energy to redefine the link weight between edges $w(u, v)$. The simulation later compares the MAFEST result with the traditional LMST algorithm. Traditional LMST algorithm only considers energy consumption of sensors as the link weight when building a local minimum spanning tree. Without consideration of energy balance, this may lead to a bottleneck of sensor rapid death due to network overload.

In this experimental environment, we build an environment with 56 sensors and each sensor has the same initial energy. Comparing two experiments, we find that the MAFEST algorithm, for topology change, has obvious advantages over the LMST algorithm in sensitivity and energy.

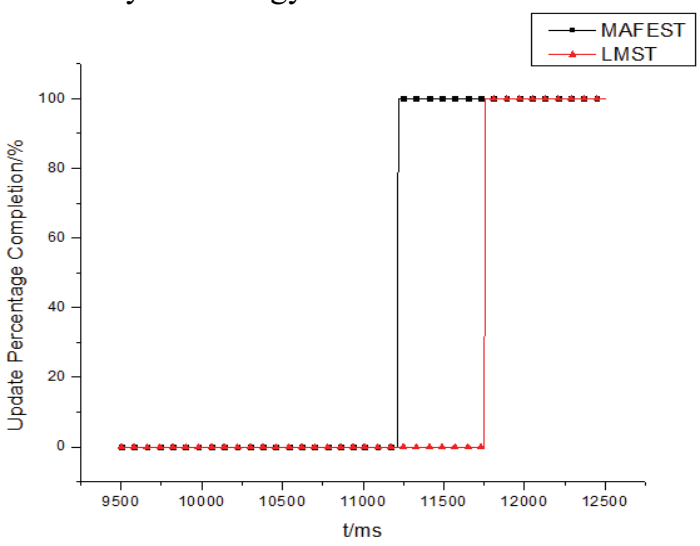

Fig. 8. (Color online) Percentage of topology update completion in sensor node observed

When 11000 milliseconds passed in this simulation, we randomly selected one sensor in the network to observe the speed of update percentage completion, after manually changing the network topology structure to make one pair of network sensors from not connect to connect. In the figure $8, \mathrm{X}$-axis represents how much time the entire network had run and $\mathrm{Y}$-axis represents the completed update percentage of the observed sensor to new network topology. It can be seen that MAFEST algorithm's speed is a bit faster than the LMST algorithm.

In figure 9, this graph describes the change of the percentage of sensors, which know the change of network topology, in all sensors of the network with time passed by. X-axis represents the whole time passed when we started this simulation and $\mathrm{Y}$-axis represents the total percentage of sensors which found the event occurred. There is a high slope in LMST and a big advantage in MAFEST. The main reason for this big advantage is that LMST algorithm updates network topology information by broadcasting HELLO packets and the broadcast radio frequency can't be too frequent 
(severe impact on the effective bandwidth usage of the network) which will slow the network topology response. The algorithm of MAFEST uses the process of mobile agent migration to update the topology information, therefore, bandwidth usage is much less than LMST algorithm and the same with the speed of getting topology information.

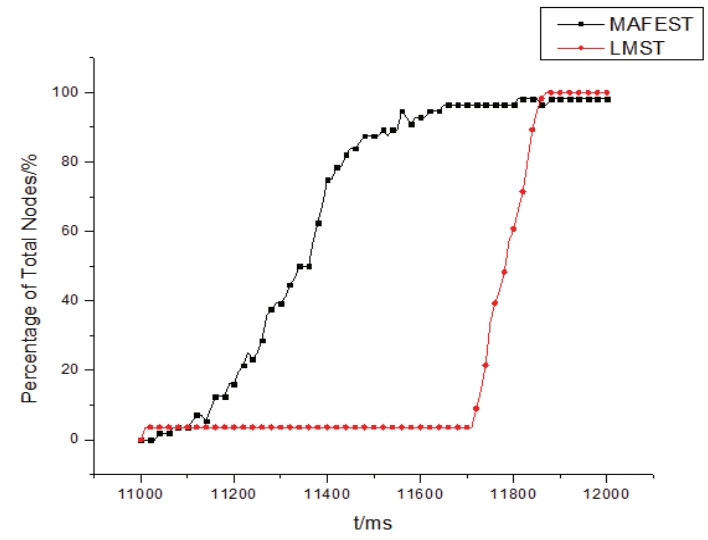

Fig. 9. (Color online) Sensor node percentage of the entire network about entire network topology gained

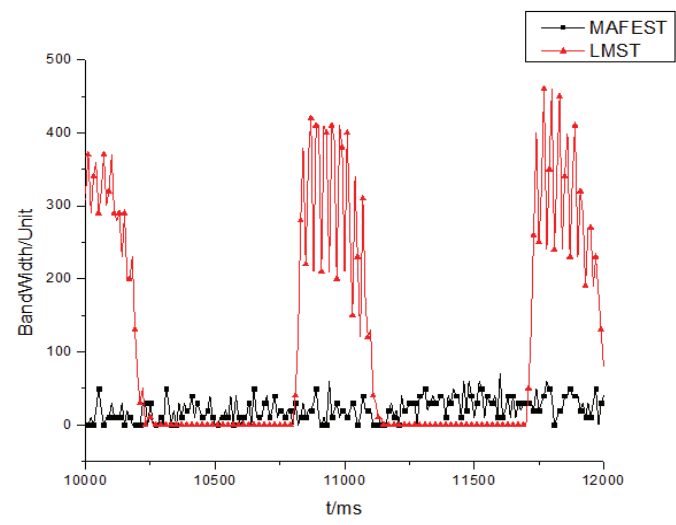

Fig. 10. (Color online) Bandwidth consumed by network topology maintenance

Figure 10 is one graph that indicates the bandwidth consumed with time passed in order to maintain the network topology in one fixed period of the simulation. $\mathrm{X}$-axis represents the time passed since simulation begins, and $\mathrm{Y}$-axis represents the network bandwidth consumed. Mobile agents cost less bandwidth which can save energy consumption of network sensor nodes implicitly.

Figure 11 shows the percentage of the remaining sensors in the network sensor with time passed by. The percentage of the remaining sensor nodes for MAFEST is higher than LMST algorithm. In LMST algorithm, the sensor nodes begin to die at the 108th seconds, while MAFEST at 130th which effectively reduces the energy consumption of the whole network.

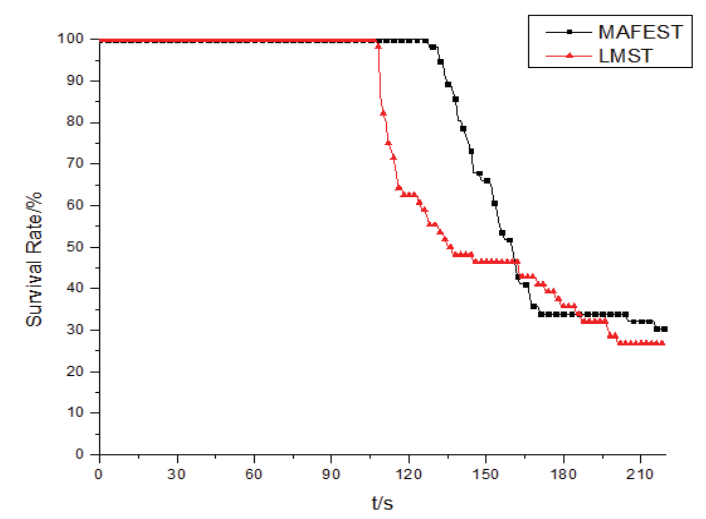

Fig. 11. (Color online) Percentage of the remaining sensor nodes

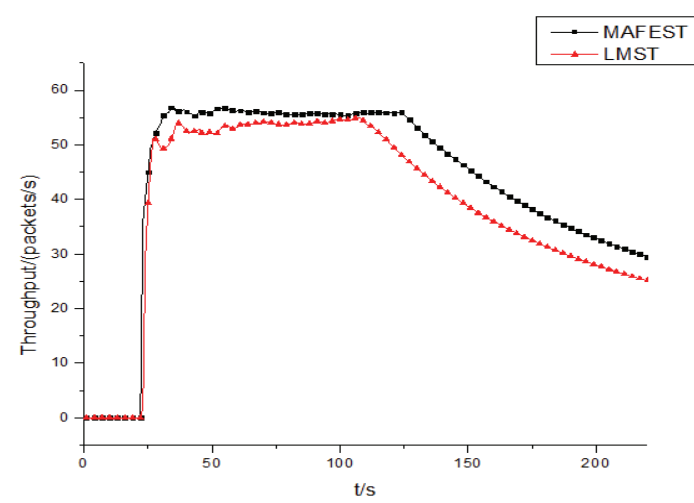

Fig. 12. (Color online) Network throughput

Throughput is the radio of the packets amount sent to destination and time consumed. This experiment started to send data packets to each other at the 22th second since simulation began and got the result in figure 12, where $\mathrm{X}$-axis represents the time passed, and $\mathrm{Y}$-axis represents the throughput. At the beginning of figure 12, the throughput of two algorithms just is straight up and reaches a stable state. Then, with sensors' energy exhausted, both algorithms' throughput decrease. LMST sensors firstly begin to die so reasonably the throughput in LMST will be the first to begin decrease.

Mailing rate is defined as the radio of total data packets received and the total data packets sent. Compared with the mailing rate of two algorithms, in figure $13 \mathrm{X}$-axis represents time passed in network, $\mathrm{Y}$ - 
axis represents the mailing rate for the entire network. The mailing rate curves of two algorithms the remained the same at the beginning, but mailing rate of LMST begins to decrease due to the early death of its sensors.

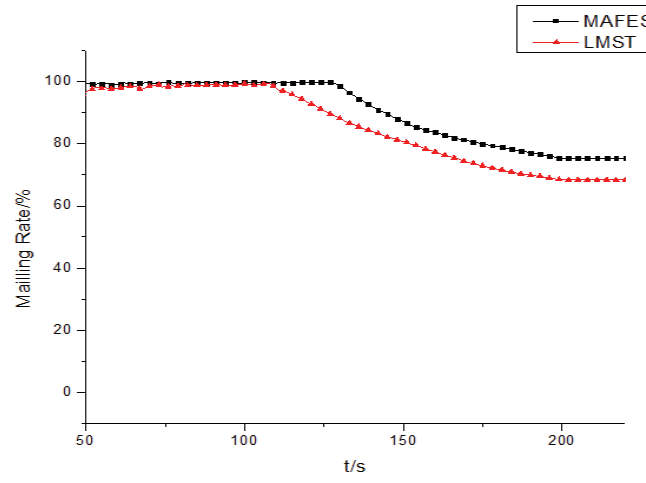

Fig. 13. (Color online) Network mailing rate

\section{The Conclusion}

This paper holds the essence of Internet of Things topology discovery and uses a robust mobile agent model which can produce proper number of agents according to different network conditions in the mobile agent network. A mobile agent can use the designed strategy to move and interact with each other in Internet of things based on wireless sensor nodes. This algorithm does not depend on the network sensor node collaboration without increasing the network burden, which meet the needs of limited bandwidth and other resources. Meanwhile, in order to extend the network lifetime, we should try to ensure that wireless sensors energy consumption is relatively balanced. The distributed topology algorithm we have proposed uses the method of adjusting transmission power of network sensors which have fuzzy energy feature.

\section{Acknowledgment}

This research is supported by the Fundamental Research Funds for the Central Universities-Project of University of Electronic Science and Technology of China with grant number ZYGX2010J075 and the Science and Technology Support Foundation of Sichuan Province with grant number 2012GZ0061, the National Natural Science Foundation of China with grant number 61300192.

\section{References}

1. Gu Jing Jing and Chen Song Can, et al. Wireless sensor network-based topology structures for the Internet of Things localization, Chinese Journal of Computers. 33(9)(2010)1548-1556.

2. Zheng Weia and LIU San yangb, et al. Dynamic mobile agent routing algorithm in sensor network, Control and Decision. 25(7)(2010)1035-1039.

3. Zhou Si Wang and Lin Ya Ping, et al. A study of trajectory-based mobile agent dynamic routes algorithm for data fusion in wireless sensor networks, Chinese Journal of Computers. 30(6)(2007) 894-904.

4. R. Ramanathan and R. Rosales-Hain. Topology control of multihop wireless networks using transmit power adjustment, in Proc. IEEE INFOCOM (Tel Aviv, Israel, Mar. 2000), pp.404-413.

5. S. Narayanaswamy and V. Kawadia, et al. Power control in ad hoc networks: theory, architecture, algorithm and implementation of the COMPOW protocol, in Proc. Eur. Wireless 2002, Next Generation Wireless Networks: Technologies, Protocols, Services and Applications (Florence, Italy, Feb. 2002), pp. 156-162.

6. V. Kawadia and P. Kumar. Power control and clustering in ad hoc networks, in Proc. IEEE INFOCOM (San Francisco, CA, Apr. 2003), pp. 459-469.

7. L. Li and J. Y. Halpern, et al. Analysis of a cone-based distributed topology control algorithm for wireless multihop networks, in Proc. ACM Symp. Principles of Distributed Computing (Newport, RI, Aug.2001), pp.264-273.

8. LI Ning and HOU J C, et al. Design and analysis of an MST-based topology control algorithm, in Proc. TwentySecond Annual Joint Conf. of the IEEE Computer and Communications Societies (Sanfrancisco, CA, INFOCOM , Jul. 2003), pp. 1702-1712.

9. WANG Min Yi and ZHOU Ming Tian, et al. Cooperative mobile agents in dynamic network environment, Journal of Computer Research and Development. 38(12)(2001)1454-1460.

10. Mao Y Y, Kschischang F R, Li B C, et al. A factor graph approach to link loss monitoring in wireless sensor networks, IEEE Journal on Selected Areas in Communications. 23(4)(2005)820-829.

11. Zhao J,Govindan R, Estrin D. Residual energy scan for monitoring sensor networks, in Proc. IEEE Wireless Communications and Networking(Orlando, FL, Mar.2002), pp.356-362.

12. Hartl G, Li B. Loss inference in wireless sensor networks based on data aggregation, in Proc. third International Symposium on Information Processing in Sensor Networks(Berkeley,CA, April 2004), pp.396-404.

13. Niu Xin Zheng and She Kun, et al. Research on fuzzy knowledge processing for routing algorithm in mobile Peer-to-Peer networks, Journal of Computer Research and Development. 45(6)( 2008) 930-941.

14. Safwan Al-Omari and Weisong Shi. A novel topology discovery service for self-organized WSNs, in 
Proc.Wireless Algorithms, Systems and Applications (Chicago, IL, Aug. 2007), pp. 213-218.

15. Abdulaziz Barnawi, Roshdy Hafez. A time \& energy efficient topology discovery and scheduling protocol for wireless sensor networks, in Proc. Computational Science and Engineering (Vancouver, BC, Aug. 2009), pp. 570578.

16. Mehwish Nasim, Yasir Fayyaz, Muhammad Younus Javed. Bounded degree energy aware topology discovery in Ad hoc wireless sensor networks, in Proc. 2009 5th Intelligent Sensors, Sensor Networks and Information Processing (Melbourne,VIC, Dec. 2009), pp.13-18.

17. Alireza A. Nezhad, Ali Miri, Dimitris Makrakis. Efficient topology discovery for multihop wireless sensor networks, in Proc.6th Annual Communication Networks and Services Research Conference(Halifax, NS, May 2008), pp.358-365.

18. Yasir Fayyaz, Mehwish Nasim, Muhammad Younus Javed. Maximal weight topology discovery in ad hoc wireless sensor networks, in Proc. 2010 10th IEEE Computer and Information Technology (Bradford, July 2010), pp. 715-722.

19. Jiliang Wang, Mo Li, Yunhao Liu. Fractured voronoi segments: topology discovery for wireless sensor networks, in Proc.2010 IEEE 7th Mobile Adhoc and Sensor Systems (San Francisco, CA, Nov. 2010), pp.137145.

20. Mingze Zhang, Mun Choon Chan and A. L. Ananda. Location-aided topology discovery for wireless sensor networks, in Proc.IEEE Communications(Beijing,China, May 2008), pp.2717-2722.

21. Jing Wei, Wei Guo, Jian Su, et al. Mobile agent based topology discovery in mobile ad hoc networks, in Proc. 5th Wireless Communications, Networking and Mobile Computing(Beijing,China, Sept. 2009), pp. 1-4.

22. Adnan Ahmed, Behrouz Far. Mobile agent system for network topology discovery, in Proc. Canadian Electrical and Computer Engineering(Ottawa,Ont, May 2006), pp. 1376-1379.

23. NIKOS MIGAS, WILLIAM J.BUCHANAN, and KEVIN A. McARTNEY. Mobile agents for routing, topology discovery, and automatic network reconfiguration in Ad-Hoc Networks, in Proc.10th IEEE International Conference and Workshop on the Engineering of Computer-Based Systems(April 2003), pp. 200-206.

24. R. Tobias Meier, Jürgen Dunkel, Yoshiaki Kakuda. Mobile agents for service discovery in ad hoc networks, in Proc.22nd Advanced Information Networking and Applications(Okinawa, March 2008), pp.114 -121.

25. Adnan Ahmed, Behrouz Far. Performance of mobile agent based network topology discovery, in Proc. Canadian Electrical and Computer Engineering(Vancouver, BC, April 2007), pp.66-69.

26. Bogdan T. Nassu, Takashi Nanya, Elias P. Duarte Jr. Topology discovery in dynamic and decentralized networks with mobile agents and swarm intelligence, in
Proc.7th Intelligent Systems Design and Applications(Rio de Janeiro, Oct. 2007), pp.685-690.

27. Adnan Ahmed and Behrouz Homayoun Far. Topology discovery for network fault management using mobile agents in Ad-hoc networks, in Proc. Canadian Electrical and Computer Engineering(Saskatoon, Sask, May 2005), pp. 2041-2044. 